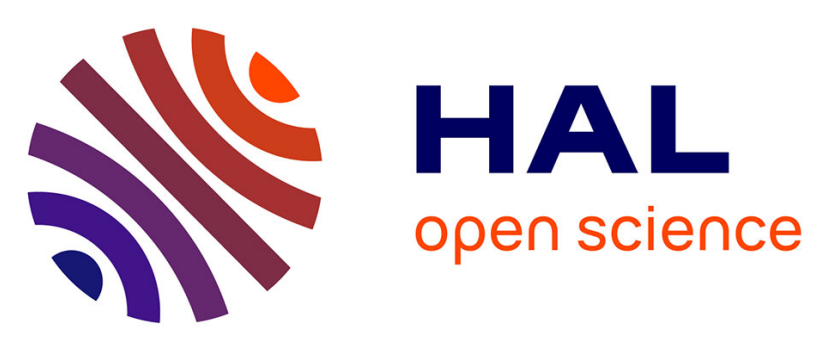

\title{
Initial Study on Fault Tolerant Control with Actuator Failure Detection for a Multi Motor Electric Vehicle
}

Bruno Dos Santos, Rui Esteves Araújo

\section{To cite this version:}

Bruno Dos Santos, Rui Esteves Araújo. Initial Study on Fault Tolerant Control with Actuator Failure Detection for a Multi Motor Electric Vehicle. 7th Doctoral Conference on Computing, Electrical and Industrial Systems (DoCEIS), Apr 2016, Costa de Caparica, Portugal. pp.197-205, 10.1007/978-3319-31165-4_20. hal-01438245

\section{HAL Id: hal-01438245 \\ https://hal.inria.fr/hal-01438245}

Submitted on 17 Jan 2017

HAL is a multi-disciplinary open access archive for the deposit and dissemination of scientific research documents, whether they are published or not. The documents may come from teaching and research institutions in France or abroad, or from public or private research centers.
L'archive ouverte pluridisciplinaire HAL, est destinée au dépôt et à la diffusion de documents scientifiques de niveau recherche, publiés ou non, émanant des établissements d'enseignement et de recherche français ou étrangers, des laboratoires publics ou privés. 


\title{
Initial Study on Fault Tolerant Control with Actuator Failure Detection for a Multi Motor Electric Vehicle
}

\author{
Bruno dos Santos, Rui Esteves Araújo \\ INESC TEC, Faculty of Engineering, University of Porto, 4200-465 Porto, PORTUGAL \\ \{bruno.laranjo, raraujo\}@fe.up.pt
}

\begin{abstract}
This study presents a scheme to detect and isolate faults in overactuated electric vehicles. Although this research work is still emerging, it already provides a view of the main challenges on the problem and discusses some possible approaches that can be useful to overcome the key difficulties. This paper intends to present a fault detection algorithm based on Unknown Input Observer (UIO). The residuals are built through the difference of signals between the measured outputs and the output estimations from the observer. The main idea is to detect fault in the electric motors and steering wheel actuator. The algorithm is presented and tested with some fault scenarios using the co-simulation tool between Simulink/MATLAB and the high-fidelity model from Carsim software.
\end{abstract}

Keywords: Fault Tolerant Control, Fault Detection and Isolation, Unknown Input Observer, Electric Vehicles.

\section{Introduction}

The road map on road transport published by ERTRAC, EPOSS and SMARTGRIDS in 2010 had the expectation that the adaptation of conventional vehicles to electric power train is the first milestone [1]. At the present stage, most compact electric vehicle is based on today's drive train platforms with one central electric motor. However the potential of novel power drive concepts is not completely achieved with these first generation vehicles. Multiple electric motors mounted close to the wheels or even in the wheel open the development of new compact vehicle drive train architectures.

System redundancy could be explored in order to have high safety and robustness requirements. The system redundancy in multi-motor electric vehicles should be exploited. If an actuator fails, it is necessary to redistribute the control effort among the healthy actuators such that stability is retained.

Some accidents are prevented by using mechanism that detect and inform the driver about a fault. Between July 2005 and December $20076.8 \%$ of all accidents are something related with some vehicle malfunction [2]. In the next section will be focused on the formulation of the Research Questions that need to be answer with this $\mathrm{PhD}$ work. 


\subsection{Research Question}

Research Question 1: How can we improve the architecture of the fault tolerant control with Fault Diagnosis for four-wheel independently driven electric vehicle?

The key idea to be explored is to replace the standard control allocation by the concept of "smart control allocation" in order to improve the overall structure. The main objective of the smart control allocation is to ensure that the system adapts in the best way when an actuator fails.

A general overview of control architecture is shown in the fig. 1 . The reconfigurable allocator uses information provided by the fault diagnosis to accommodate faults by performing an appropriate reconfiguration. The fault diagnosis system uses the signals from the Hardware and IMU (Inertial Measurement Unit) sensors with Unknown Input Observer (UIO) algorithm to detect faults. The fault diagnosis system output will be a set of fault flags. This information will be interpreted as the total fault indicator vector, which will be an input for the reconfigurable allocator. The relationships between actuators states, fault type and remedial actions will be defined in the fault code table in order to specify the constraint bounds of the control allocation algorithm.

Research Question 2: How should we exploit the fault tolerant detection subsystem to implement an active Fault Tolerant Control (FTC) for multi-motor electric vehicles?

If we started by investigating how the use of the information provided by the Fault Detection (FD) scheme, we could help to improve the active FTC performance. One of the biggest challenges of this thesis is the FD capability to send useful information to the controller. To be useful, the information sent to the controller has to be accurate and needs to be on time. Although some losses in accuracy can be compensated by using robust control techniques, also the delay issues will be crucial. The response time or detection test time needs to be the slowest possible in order to maximize the fault reaction time.

\section{Contribution to Cyber-Physical Systems (CPS)}

From the automotive electronic point of view, the modern vehicles contain a large number of CPS's such as Anti-Lock Brake Systems, Traction Control, Electronic Stability Program, etc. The same happen for the electric vehicles. The tight interaction between all the computational devices like the battery management system, electric drives of motors and the physical components brings many challenges in this field. For safety and efficiency reasons, these spatially distributed components require a sophisticated monitoring and control. For this purpose, a paradigm change for Electrical and Electronic (E/E) architectures becomes necessary to increase the flexibility between elements [3].

Functional safety is taking more importance in electric vehicles development, due the increased complexity and the wide employment of electrical and electronic systems. In contrast with the past, it will be more important for automotive industry to 


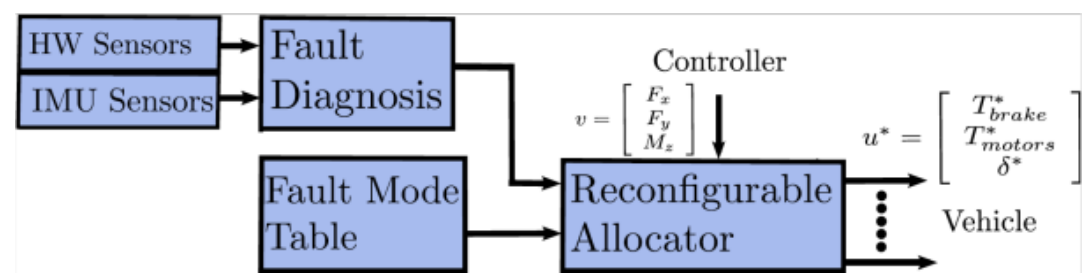

Fig 1 - Proposed FTC structure in the PhD.

put in place a normative process to assure the reliability of the safety-related systems, as opposed to regulate themselves with different rules [4].

In order to ensure functional safety the automotive functional safety standard was launched (ISO 26262) [5] to solve the problem of components faults in electrical and electronic system in vehicles. This standard is an adaptation of the IEC 61508 and overcome all the lifecycle of electrical/electronic components. Some metrics introduced by the norm can be useful to quantify the fault tolerant control performance.

\section{State of the Art}

The literature presents numerous FDI applications for aeronautical and aero-space systems, chemical process, nuclear plants, power system, automotive and electronic systems, see for example [6] or [7]. Also a FDI for the ROboMObil, a concept car, was designed and tested in real operating conditions on DLR (Deutschen Zentrums für Luft-und Raumfahrt) institute. They used the well-known parity equation to detect faults in sensors and actuators [8]. Also, the work started by Rongrong Wang in his $\mathrm{PhD}$ thesis at Ohio State University [9] demonstrates the significance of having a FDI scheme to the same type of vehicles.

Only few papers about fault tolerant control of over actuated electric vehicles consider the fault diagnosis in their studies, others assuming that the faults are perfectly identified [10]. However, this is not a realistic hypothesis since fault diagnosis is a very challenging topic, especially for non-linear systems.

In this paper is presented an FDI system based on a Linear Parameter Varying (LPV) UIO scheme. The idea of this approach is to represent the nonlinearities inherent in the model by a LPV approach. Then, the way to use the proposed LPV model is to develop a bank of UIOs that generates a set of residuals which will be sensitive to a pre-established set of faults while insensitive to the others which are considered as disturbances. 


\section{Vehicle Model}

The vehicle modeling is composed by several physical quantities represented in a three coordinate system. The dynamics behavior is based on Euler and Newton laws of motion. The system axis $(x, y)$ is fixed in the vehicle Center of Gravity $(\mathrm{CoG})$, and the movement is based on CoG's velocities and forces:

$$
\dot{v}_{x}=v_{y} r+\frac{F_{x}}{m}, \quad \dot{v}_{y}=-v_{x} r+\frac{F_{y}}{m}, \quad \dot{r}=\frac{M_{z}}{I_{z}}
$$

Where $v_{x}$ is the longitudinal speed, $v_{y}$ is the lateral speed and $r$ is the yaw rate. Through a simplified Euler-Newton equation system it is possible to represent the vehicle behavior as function of the longitudinal force $F_{x}$ the lateral force $F_{y}$ and the yaw moment $M_{z}$ applied in the CoG. These virtual forces are the forces that act to move the vehicle. The virtual inputs are related width the tire forces by:

$$
\begin{aligned}
& v=\left[\begin{array}{lll}
F_{x} & F_{y} & M_{z}
\end{array}\right], \quad v=B F_{x y} \\
& v=\left[\begin{array}{cccccccc}
1 & 0 & 1 & 0 & 1 & 0 & 1 & 0 \\
0 & 1 & 0 & 1 & 0 & 1 & 0 & 1 \\
-\frac{l_{s}}{2} & l_{f} & \frac{l_{s}}{2} & l_{f} & -\frac{l_{s}}{2} & -l_{r} & \frac{l_{s}}{2} & -l_{r}
\end{array}\right] F_{x y} \\
& F_{x y}=\left[\begin{array}{llllllll}
F_{x F L} & F_{y F L} & F_{x F R} & F_{y F R} & F_{x R L} & F_{y R L} & F_{x R R} & F_{y R R}
\end{array}\right] \\
& {\left[\begin{array}{ll}
F_{x i} & F_{y i}
\end{array}\right]=\left[\begin{array}{cc}
\cos \left(\delta_{i}\right) & -\sin \left(\delta_{i}\right) \\
\sin \left(\delta_{i}\right) & \cos \left(\delta_{i}\right)
\end{array}\right]\left[\begin{array}{c}
F_{c i} \\
F_{l i}
\end{array}\right], i \in\{F L, F R, R L, R R\}}
\end{aligned}
$$

Where $F_{c i}$ and $F_{l i}$ are the centripetal and longitudinal tire forces applied to the ground. In order to define the tire forces, the Pacjeka semi empiric model can be employed [11]. In this section we assume that the forces are in the linear part of the tire function and also that the side slip angle for each tire is small and the difference between the torque input and the longitudinal force is zero $\left(F_{l i}-T_{i} r_{i} \approx 0\right)$. At this stage of the work we assume that the torque signal send to the in-wheel actuator is a combination of the motor and brake system signals. So the tire force components are:

$$
F_{c i_{\text {front }}}=N_{i} \cdot D_{i}\left(\delta_{i}-\frac{v_{y}-l_{f} r}{v_{x}}\right), F_{c i_{\text {rear }}}=N_{i} \cdot D_{i}\left(\frac{v_{y}-l_{r} r}{v_{x}}\right) \quad F_{l i}=\frac{T_{i}}{R}
$$

where $T_{i}$ is the input torque to each in-wheel motor, $\delta_{i}$ is the steering angle in each wheel ( $\delta_{i}=0$ for the rear wheels and for the front wheels the steering angle is equal to the command input). Also $N_{i}$ is the normal force applied in the wheel; $D_{i}$ is the cornering stiffness; $l_{f}$ and $l_{r}$ are the distance from the CoG to the front and rear axis. 


\section{Fault Detection System through LPV UIO}

In order to detect actuator faults, the command inputs from the driver are sent to the force observer. The virtual force observer is obtained applying the equations $(1,2,3)$. This estimator determine the value of each virtual forces that are delivered to the vehicle. As a result, the state reconstruction is carried out by a bank of UIOs using the virtual force provided by the force observer with different outputs. With the purpose to achieve isolation of faults, the inputs (individual virtual forces) need to be divided into groups and selected the output related with the fault in consideration.

\subsection{LPV UIO}

In this work, it is considered that only one actuator fault is detected by each UIO. More details about the UIO observer can be found in [12]. For a LPV system like:

$$
\left\{\begin{array}{c}
\dot{x}(t)=A(\rho) x(t)+B(\rho) u(t)+D(\rho) d(t)+B_{s}(\rho) f_{s}(t) \\
y(t)=C x(t)
\end{array}\right.
$$

where $x(t) \in \mathbb{R}^{\mathrm{n}} ; u(t) \in \mathbb{R}^{\mathrm{m}} ; d(t) \in \mathbb{R}^{\mathrm{d}} ; y(t) \in \mathbb{R}^{\mathrm{p}}$; and $f_{S}(t) \in \mathbb{R}^{1}$; are the state vector, input vector, perturbation vector, output vector and fault vector, respectively. The distribution matrix for the fault is represented by the corresponding column number $\left(s^{t h}\right)$ of $B\left(B_{S}\right)$. The parameter $\rho(t)=\left[\rho_{1}, \ldots, \rho_{r}\right] \in \mathbb{D}$ is a time varying function. The requirements to LPV system are: all the matrices are affine in $\rho$; the parameter $\rho$ is accessible; as well as the $\rho$ and $\rho$ are bounded. We are going to first transform the system into is polytopic form:

$$
A(\rho)=\sum_{i=1}^{v} h_{i}(\rho(t)) A_{i}, \text { with: } h_{i}(\rho)>0 \text { and } \sum_{i=1}^{r} h_{i}(\rho(t))=1
$$

where $v$ is the number of vertices limits of $\rho$. The new system is described by:

$$
\left\{\begin{array}{c}
\dot{x}(t)=\sum_{i=1}^{v} h_{i}(\rho)\left(A_{i} x+B_{i} u+D_{i} d+B_{s i} f_{s}\right) \\
y(t)=C x
\end{array}\right.
$$

This structure allows the usage of linear UIO for the transformed system:

$$
\left\{\begin{array}{c}
z=\sum_{i=1}^{v} h_{i}(\rho)\left(F_{i} z+G_{i} v+K_{i} y\right) \\
\hat{x}=z-H y
\end{array}\right.
$$


The goal is that estimation error $(e(t)=x(t)-\hat{x}(t))$ converges to zero under any initial conditions and any input. Replacing the estimate error expression from eq (7), it follows that $e=T x-z$, where $T=I-H C$ (note that the index $i$ is omitted). Solving this equation for the time derivative of the error gives:

$$
\begin{gathered}
e=\begin{array}{c}
F e+(T B-G) u+(T D) d+ \\
+\left(T B_{s}\right) f_{s}+(T A-K C+F H C-F) x
\end{array}
\end{gathered}
$$

In order to design the UIO, is necessary to meet the conditions:

$$
\begin{array}{cc}
\text { (C1) } G=T B(\text { cancelu }(t)) ; & (C 2) T D=0(\text { canceld }(t)) \\
\text { (C3) } F=T A-L C \text { (F is "Hurwitz"); } & \text { (C4) } K=L-F H \\
\text { (C5) } \operatorname{rank}\left(T B_{S}\right)=\operatorname{rank}\left(B_{S}\right) & \text { (C6) } T=I-H C
\end{array}
$$

Thus, if all conditions are respected the UIO error dynamics becomes $\dot{\mathrm{e}}=\mathrm{Fe}+T B_{s} f_{s}$ and the residual correspond to $r=C e(t)$.

For the disturbance decoupling, first it is necessary to check if $\operatorname{rank}(C D)=\operatorname{rank}(D)$ to guaranteed a solution for the system $H C D=D$ (from the condition (C2) and (C6)). One generalized solution for $H$ can be expressed by [13]:

$$
H=D(C D)^{+}+H_{0}\left(I-(C D)(C D)^{+}\right)
$$

where $\left(X^{+}\right)$is the generalized inverse matrix given by $(X)^{+}=\left((X)^{T}(X)^{-1}\right)(X)^{T}$, since $X$ is full rank. $H_{0}$ is arbitrary matrix of appropriate dimensions and give some freedom on the design. The eigenvalues of $F$ can be arbitrary located by choosing a suitable matrix $L$, only if the pair $(T A, C)$ is observable. For LPV systems the condition $\left(\operatorname{rank}\left(C D_{i}\right)=\operatorname{rank}\left(D_{i}\right)\right)$ for the linear decoupling is not sufficient, it is necessary that $\left.\left(\operatorname{rank}\left(C\left[D_{1}, \ldots, D_{v}\right]\right)=\operatorname{rank}\left[D_{1}, \ldots, D_{v}\right]\right)\right)$ in order of to obtain $\left(H C D_{\rho}=D_{\rho}\right)$. Also the polytopic stability of $\left(L_{i}\right)$ is subject to the global stability of $F=T A_{\rho}-L_{\rho} C$ where $\left(X_{\rho}\right)$ are all matrices from the "LPV space").

UIO design - The system in eq. (1) can be written into a LPV for the $(x, y)$ dynamics. The LPV system has following parameters:

$$
x=\left[v_{x}, v_{y}\right]^{T} ; \quad \rho=r ; \quad A_{\rho}=\left[\begin{array}{cc}
0 & \rho \\
-\rho & 0
\end{array}\right] ; \quad B_{\rho}=\frac{1}{m} I_{2} ; \quad C=I_{2} ; \quad u=\left[F_{x}, F_{y}\right]
$$

The residual in yaw rate dynamics is the difference between the models in a nonfault scenario with the read values. The UIO algorithm design is presented in Table 1. 


\subsection{Simulations}

The proposed LPV-UIO scheme has been simulated in different case scenarios. The nonlinear vehicle model was simulated through the Carsim software in co-simulation with Simulink/MATLAB where the proposed observer was implemented. The vehicle parameters that have been used to perform simulations are on the table 2 .

In order to demonstrate the capacity to detect faults in actuators it is defined two fault scenarios: The first, a J-turn maneuver with steer fault in constant acceleration with faults in two of the four motors. In this simulations only abrupt fault are considered (step). The fault amplitude is for the steering test a part of the steering input. For the wheel test it is always considered the total failure of the motor in-wheel unit. The inputs of torque to wheel and steering angle reference from the driver are the system input.In the simulations, when a fault occurs, the same fault has to be signalized by an alteration in the residual for each component. The residual amplitude has to be greater than a certain threshold in order to signalize that there is a fault.

Steer Fault - The vehicle starts with an initial longitudinal speed of $20 \mathrm{~m} / \mathrm{s}$ with no steer input. At the time $t=1 \mathrm{~s}$ the steering angle is set in $0.05 \mathrm{rad}$. This steering is kept constant for the entire test. In this simulation, several faults input have been tested. From the Fig. 2 it is possible to see that the vehicle global trajectory is affected by the steering fault. At the moment when steering input is set to $0.05 \mathrm{rad}$, the residuals have a slightly change that are negated by the observer. When at $t=5 \mathrm{~s}$ the fault are injected in the system, all residuals reflects the actuator faults. Obviously the steering fault impact has more influence in the residual provided by the second UIO and from $M_{z}$. The steering component has more impact in these residuals.

Table 1 - UIO Algorithm design for planar motion;

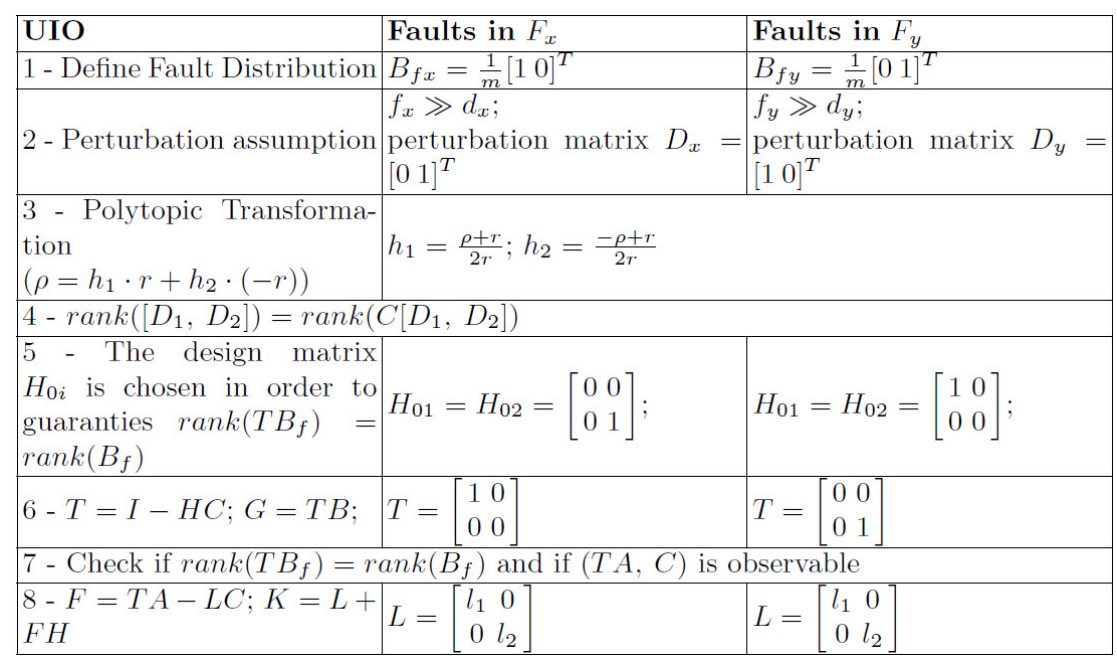


Table 2 - Vehicle Parameter used in the simulations

\begin{tabular}{|l|c|c|l|c|c|}
\hline Parameters & Symbol & Value & Parameters & Symbol & Value \\
\hline vehicle total mass & $m$ & $1100 \mathrm{~kg}$ & wheel radius & $R$ & $0.3 \mathrm{~m}$ \\
\hline tire cornering stiffness & $D$ & $0.2179 \mathrm{rad}^{-1}$ & wheel moment of inertia & $I_{w}$ & $1.1 \mathrm{kgm}^{2}$ \\
\hline $\begin{array}{l}\text { distance from CoG to front } \\
\text { axle }\end{array}$ & $l_{f}$ & $1.2 \mathrm{~m}$ & $\begin{array}{l}\text { distance from CoG to rear } \\
\text { axle }\end{array}$ & $l_{r}$ & $1.3 \mathrm{~m}$ \\
\hline $\begin{array}{l}\text { distance from CoG to } \\
\text { ground }\end{array}$ & $h$ & $0.37 \mathrm{~m}$ & track width & $l_{s}$ & $1.5 \mathrm{~m}$ \\
\hline $\begin{array}{l}\text { moment of inertia about } \\
\text { vertical axis }\end{array}$ & $I_{z}$ & $996 \mathrm{kgm}^{2}$ & & & \\
\hline
\end{tabular}

In-wheel Motor Faults - In the second test, the vehicle starts with equal initial speed of the first test $v_{x}=20 \mathrm{~m} / \mathrm{s}$. In this case the steering remains $\delta=0^{\circ} \mathrm{rad}$ for the entire test. At $\mathrm{t}=5 \mathrm{~s}$ in-wheel motor faults combinations are injected in the system. The residuals for this test are shown in the Fig 3. The first point to notice is that the faults in wheels are signalized with low amplitude signals. It is because the faults have low contribution to the trajectory. Also, it is necessary to point out that the observer gain is very low, approximately $10^{-3}$. However the trajectory deviation is not large. Also, in the residual in $F_{x}$ the value in a non-fault case is not zero due to the longitudinal friction forces that were not modeled in Equation (1).

\section{Conclusions and Future Work}

This work considers the design of UIO for use in fault detection for an over-actuated electric vehicle. Although research work is in its first steps, it already provides an overview on the problem while discussing some possible approaches and proposes a method to overcome the key difficulties. Simulation results show a good performance in order to detect several types of faults. Future work, currently under way, includes find a way that does not use the information about lateral velocity as well as implement a validation of the proposed scheme on the prototype vehicle.

Acknowledgments. This work is partially funded by National Funds through FCT Science and Technology Foundation, through the scholarships SFRH/BD/90418/2012. 


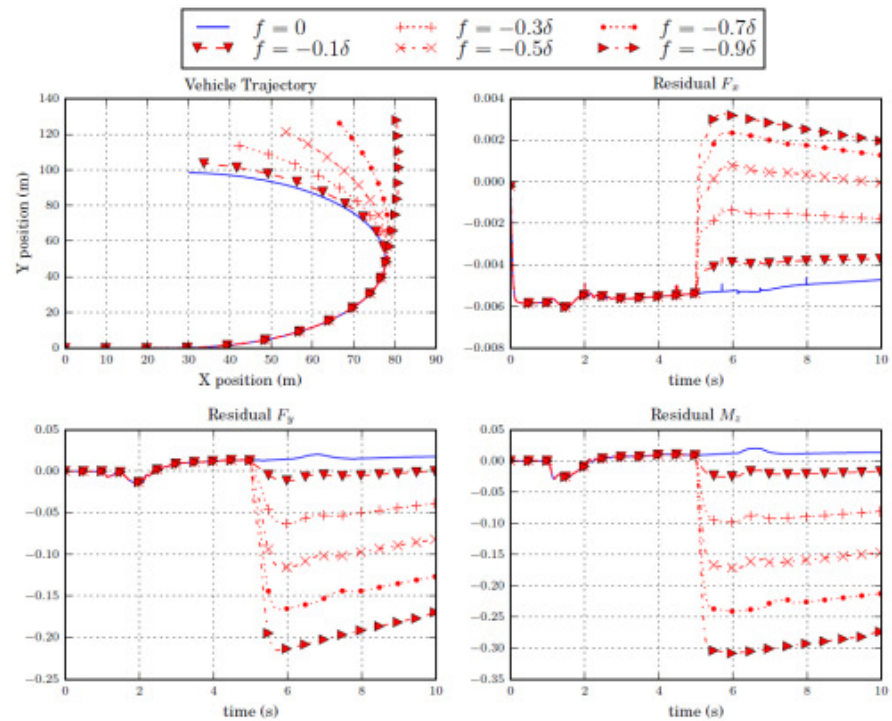

Fig 2 - Results for the steer fault test.

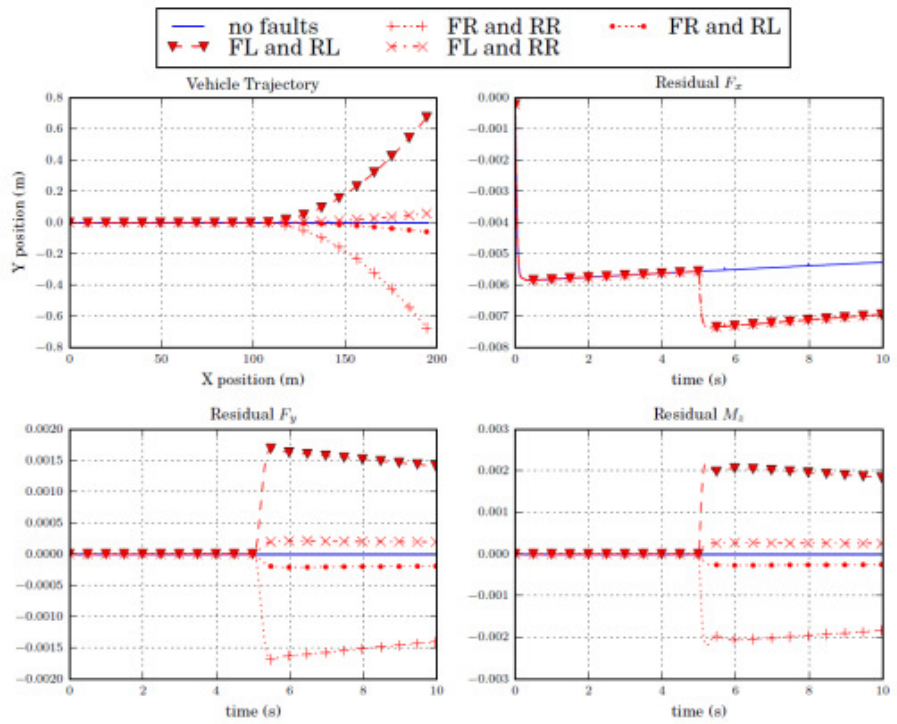

Fig 3 - Results for the in-wheel motor fault test. 


\section{References}

1. Ertrac, EPoSS, SmartGrids: European Roadmap Electrification of Road Transport. (2010).

2. NHTSA: National Motor Vehicle Crash Causation Survey Report to Congress. (2008).

3. Sankavaram, C., Kodali, A., Pattipati, K.R.: An integrated health management process for automotive cyber-physical systems. Int. Conf. Comput. Netw. Commun. \{ICNC\} 2013, San Diego, CA, USA, January 28-31, 2013. 82-86 (2013).

4. Boules, N.: Reinventing the Automobile: The Cyber-Physical Challenge. (2008).

5. ISO: ISO/DIS 26262 - Road vehicles - Functional safety. International Organization for Standardization / Technical Committee 22 (ISO/TC 22), Geneva, Switzerland (2011).

6. Isermann, R.: Model-based fault-detection and diagnosis - status and applications. Annu. Rev. Control. 29, 71-85 (2005).

7. Zhang, Y., Jiang, J.: Bibliographical review on reconfigurable fault-tolerant control systems. Annu. Rev. Control. 32, 229-252 (2008).

8. Ho, L.M., Ossmann, D.: Fault Detection and Isolation of Vehicle Dynamics Sensors and Actuators for an Overactuated X-by-Wire Vehicle. 6560-6566 (2014).

9. Wang, R.: Fault-Tolerant Control and Fault-Diagnosis Design for Over-Actuated Systems with Applications to Electric Ground Vehicles, (2013).

10. Lopes, A., Araujo, R.E.: Fault-tolerant control based on sliding mode for overactuated electric vehicles, (2014).

11. Pacejka, H.: Tire and Vehicle Dynamics. Elsevier Science (2012).

12. Ichalal, D., Mammar, S.: On Unknown Input Observers for LPV Systems. IEEE Trans. Ind. Electron. 62, 5870-5880 (2015).

13. Darouach, M., Zasadzinski, M., Xu, S.J.: Full-order observers for linear systems with unknown inputs. IEEE Trans. Automat. Contr. 39, 606-609 (1994). 